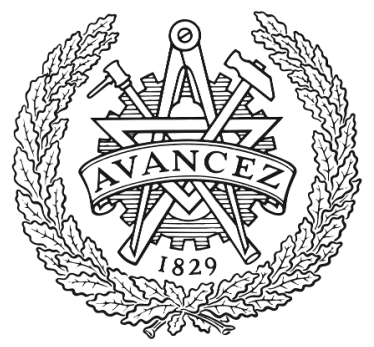

CHALMERS

UNIVERSITY OF TECHNOLOGY

\title{
Low-Complexity Accurate Mmwave Positioning for Single-Antenna Users Based on Angle-of-Departure and Adaptive Beamforming
}

Downloaded from: https://research.chalmers.se, 2023-04-26 11:02 UTC

Citation for the original published paper (version of record):

Fascista, A., Coluccia, A., Wymeersch, H. et al (2020). Low-Complexity Accurate Mmwave Positioning for Single-Antenna Users Based on Angle-of-Departure and Adaptive Beamforming. ICASSP, IEEE International Conference on Acoustics, Speech and Signal Processing - Proceedings, 2020-May: 4866-4870.

http://dx.doi.org/10.1109/ICASSP40776.2020.9053493

N.B. When citing this work, cite the original published paper. 


\title{
LOW-COMPLEXITY ACCURATE MMWAVE POSITIONING FOR SINGLE-ANTENNA USERS BASED ON ANGLE-OF-DEPARTURE AND ADAPTIVE BEAMFORMING
}

\author{
Alessio Fascista*, Angelo Coluccia*, Henk Wymeersch ${ }^{\dagger}$, Gonzalo Seco-Granados ${ }^{\S}$ \\ *Department of Innovation Engineering, University of Salento, Italy \\ ${ }^{\dagger}$ Department of Electrical Engineering, Chalmers University of Technology, Sweden \\ ${ }^{\S}$ Department of Telecommunications and Systems Engineering, Universitat Autonoma de Barcelona, Spain
}

\begin{abstract}
The problem of position estimation of a mobile user equipped with a single antenna receiver using downlink transmissions is addressed. The advantages of this setup compared to the classical MIMO and uplink scenarios are analyzed in terms of achievable theoretical performance (Cramér-Rao bounds) considering a realistic power budget. Based on this analysis, a low-complexity two-step algorithm with improved localization performance is proposed, which first performs a (coarse) angle of departure estimation and then precodes the downlink signal to introduce beamforming towards the user direction. Results demonstrate that position estimation in downlink can be potentially much more accurate than in uplink, even in presence of multiple users in the system.
\end{abstract}

Index Terms - position estimation, downlink, angle of departure (AOD), mmWave, 5G cellular networks

\section{INTRODUCTION}

Millimeter-wave (mmWave) and massive multiple-input multiple-output (MIMO) are considered key enablers for future wireless systems, including 5G cellular networks. Besides high data rates, they also allow for very accurate localization of mobile stations (MSs) thanks to much larger bandwidth and highly directional communications [1, 2]. Compared to previous-generation systems, this brings new possibilities [3]: together with the time-of-flight (TOF) measurements [4] that are also possible with traditional SISO systems, angular measurements can be also performed and represent a very useful source of information for positioning. However, in the near future, before full-fledged MIMO technology will be ready, it is expected that a large number of antennas will be available only on the base station (BS) side, i.e., a multiple-input single-output (MISO) setup.

G. Seco-Granados was supported in part by the Spanish Ministry of Science, Innovations and Universities through projects TEC2017-89925-R and TEC2017-90808-REDT.

H. Wymeersch was supported by the Swedish Research Council (VR) under project no. 2018-03701.
In general, the most direct way to obtain accurate angular measurements is using the angle-of-arrival (AOA) [5, 6]; however, in a MISO system with the array on the BS, this implies the use of uplink (UL) transmissions. This may present some drawbacks. First, the power budget in UL is generally much more unfavorable than in downlink (DL), thus resulting in poorer signal-to-noise ratio (SNR). Second, the processing load is put on the BS, which can be problematic in future $5 \mathrm{G}$ networks due to the expected massive number of low-power devices (smartphones, smart watches, etc.) that need to transmit to the BS under the IoT paradigm [7, 8]. Therefore, an interesting alternative is to perform angular measurements in DL, which implies the estimation of the angle-of-departure (AOD). In [9], the fundamental limits of position estimation for UL and DL in mmWave MIMO systems have been investigated, and a compressed-sensing algorithm that jointly exploits AOD, AOA, and TOF has been proposed in [10].

In this paper, we provide a two-fold contribution to the problem of estimating the MS position using DL transmission under a MISO system setup. First, we carry out a deeper theoretical comparison between UL and DL positioning, complementing the preliminary analysis provided in [11] with a precise assessment of the achievable performance when a realistic power budget for the UL is considered, also taking into account the presence of other users. Then, we propose an adaptive design of the transmit beamforming matrix, exploiting a (possibly coarse) knowledge of the direction where the MS is located. The theoretical analysis in terms of CramérRao bound and numerical results together demonstrate that position estimation in DL can be potentially much more accurate than in UL, even in presence of multiple users in the system. As a second contribution, we propose a practical lowcomplexity algorithm that leverages the insights derived from the analyses to achieve improved localization performance.

\section{SYSTEM MODEL}

We consider a MISO system with a BS equipped with $N_{\text {BS }}$ antennas and a MS equipped with a single antenna. We focus on a $2 \mathrm{D}$ scenario, where the BS is located in the origin, and we denote by $\boldsymbol{p}=\left[\begin{array}{ll}p_{x} & p_{y}\end{array}\right]^{T}$ the unknown MS position. 
The BS communicates by transmitting orthogonal frequency division multiplexing (OFDM) signals over $N$ different subcarriers. Particularly, $G$ signals are sequentially transmitted over time, with the $g$-th transmission comprising $M$ different symbols for each subcarrier $n=0, \ldots, N-1$, i.e., $\boldsymbol{x}^{g}[n]=$ $\left[x_{1}[n] \cdots x_{M}[n]\right]^{T} \in \mathbb{C}^{M \times 1}$. Symbols are precoded through the beamforming matrix $\boldsymbol{F}=\left[\boldsymbol{f}_{1}, \boldsymbol{f}_{2}, \ldots, \boldsymbol{f}_{M}\right] \in \mathbb{C}^{N_{\mathrm{BS}} \times M}$, with $\|\boldsymbol{F}\|_{\mathrm{F}}=1$, then transmitted over subcarrier $n$ at time slot $g$ as $\boldsymbol{s}^{g}[n]=\boldsymbol{F} \boldsymbol{x}^{g}[n], g=1, \ldots, G$.

We focus on the LOS-only link between the BS and the MS. Assuming a narrowband communication using bandwidth $B$ and a carrier frequency $f_{c}$, and that a preliminary synchronization step has been performed to compensate for the unknown clock offset $[12,13]$, the complex $1 \times N_{\text {BS }}$ channel vector at subcarrier $n$ can be expressed as

$$
\boldsymbol{h}^{T}[n]=\sqrt{N_{\mathrm{BS}}} \alpha \mathrm{e}^{\frac{-j 2 \pi n \tau}{N T_{S}}} \boldsymbol{a}_{\mathrm{BS}}^{\mathrm{H}}(\theta)
$$

where $T_{s}=1 / B$ is the sampling period, $\alpha=h / \sqrt{\rho}$ with $h$ complex channel gain and $\rho$ denoting the path loss. We will denote by $r$ and $\phi$ the modulus and phase of $\alpha$, while $\tau$ and $\theta$ denote the TOF and AOD of the LOS link, respectively. Without loss of generality, we consider a uniform linear array (ULA) with isotropic antennas, having steering vector $\boldsymbol{a}_{\mathrm{BS}}(\theta)=\frac{1}{\sqrt{N_{\mathrm{BS}}}}\left[1 e^{j \frac{2 \pi}{\lambda_{c}} d \sin \theta} \cdots e^{j\left(N_{\mathrm{BS}}-1\right) \frac{2 \pi}{\lambda_{c}} d \sin \theta}\right]^{\mathrm{T}}$ with $\lambda_{c}=c / f_{c}$ and $d=\lambda_{c} / 2$. The received signal related to the $g$-th transmission over subcarrier $n$ is given by

$$
y^{g}[n]=\sqrt{P} \boldsymbol{h}^{\mathrm{T}}[n] \boldsymbol{F} \boldsymbol{x}^{g}[n]+\nu^{g}[n]
$$

with $P$ transmit power and $\nu^{g}[n]$ circularly complex Gaussian noise having zero mean and variance $\sigma^{2}$. Without loss of generality, we assume $\sigma^{2}$ is known.

\section{DOWNLINK VS UPLINK LOCALIZATION: THEORETICAL ANALYSIS}

\subsection{Bounds for Single-Antenna User}

For the sake of the analysis, we rewrite in a different form the Fisher information matrix (FIM) and the Cramér-Rao Lower bound (CRLB) for UL and DL positioning in mmWave in the case of single-antenna users. We define the vector of unknown channel parameters as $\boldsymbol{\psi}=[r \phi \theta \tau]^{\mathrm{T}} \in \mathbb{R}^{4 \times 1}\left(\alpha=r \mathrm{e}^{j \phi}\right)$. Then, the FIM in the position domain for both UL and DL is

$$
\boldsymbol{J}_{\boldsymbol{\eta}}^{\mathrm{UL} / \mathrm{DL}}=2 \frac{P}{\sigma^{2}} \boldsymbol{T} \sum_{g=1}^{G} \sum_{n=0}^{N-1} \Re\left\{\nabla(\cdot) \nabla^{\mathrm{H}}(\cdot)\right\} \boldsymbol{T}^{\mathrm{T}}
$$

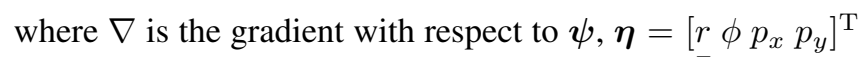
is the vector of location parameters, and $\boldsymbol{T} \stackrel{\text { def }}{=} \frac{\partial \boldsymbol{\psi}^{\mathrm{T}}}{\partial \boldsymbol{\eta}}$ is a $4 \times 4$ transformation matrix (see [11] for explicit calculation of its entries). The factor $P / \sigma^{2}$ has clearly the meaning of a SNR. The argument $m^{g}[n]$ of the gradient for DL can be obtained by inserting (1) into the noise-free version of (2), while for the UL the argument $\boldsymbol{m}^{g}[n]$ can be found in [9]:

$$
\left\{\begin{array}{ll}
m^{g}[n]=\sqrt{P N_{\mathrm{BS}}} \alpha \exp \left(\frac{-j 2 \pi n \tau}{N T_{s}}\right) \boldsymbol{a}_{\mathrm{BS}}^{\mathrm{H}}(\theta) \boldsymbol{F} \boldsymbol{x}^{g}[n] & \text { for DL } \\
\boldsymbol{m}^{g}[n]=\sqrt{P N_{\mathrm{BS}}} \alpha \exp \left(\frac{-j 2 \pi n \tau}{N T_{s}}\right) \boldsymbol{a}_{\mathrm{BS}}(\theta) x^{g}[n] & \text { for UL }
\end{array} .\right.
$$

The gradient for the DL can be easily obtained as

$$
\nabla m^{g}[n]=\sqrt{P N_{\mathrm{BS}}} \mathrm{e}^{j \phi} \mathrm{e}^{\frac{-j 2 \pi n \tau}{N T_{\mathcal{S}}}}\left[\begin{array}{c}
\boldsymbol{a}_{\mathrm{BS}}^{\mathrm{H}}(\theta) \boldsymbol{F} \boldsymbol{x}^{g}[n] \\
j r \boldsymbol{a}_{\mathrm{BS}}^{\mathrm{H}}(\theta) \boldsymbol{F} \boldsymbol{x}^{g}[n] \\
\frac{-j 2 \pi r d \cos \theta}{\lambda \lambda_{\mathrm{BS}}} \boldsymbol{a}_{\mathrm{BS}}^{\mathrm{H}}(\theta) \boldsymbol{B} \boldsymbol{F} \boldsymbol{x}^{g}[n] \\
\frac{-j 2 \pi n}{N T_{s}} r \boldsymbol{a}_{\mathrm{BS}}^{\mathrm{H}}(\theta) \boldsymbol{F} \boldsymbol{x}^{g}[n]
\end{array}\right]
$$

where $\boldsymbol{B}=\operatorname{diag}\left[\begin{array}{lll}0 & 1 & \cdots\end{array}\left(N_{\mathrm{BS}}-1\right)\right]$, while for the UL

$$
\nabla \boldsymbol{m}^{g}[n]=\sqrt{P N_{\mathrm{BS}}} \mathrm{e}^{j \phi} \mathrm{e}^{\frac{-j 2 \pi n \tau}{N T_{s}}}\left[\begin{array}{c}
\boldsymbol{a}_{\mathrm{BS}}^{\mathrm{H}}(\theta) x^{g}[n] \\
j r \boldsymbol{\boldsymbol { a } _ { \mathrm { BS } } ^ { \mathrm { H } }}(\theta) x^{g}[n] \\
\frac{-j 2 \pi r d \cos \theta}{\lambda_{c}} \boldsymbol{a}_{\mathrm{BS}}^{\mathrm{H}}(\theta) \boldsymbol{B} x^{g}[n] \\
\frac{-j 2 \pi n}{N T_{s}} r \boldsymbol{a}_{\mathrm{BS}}^{\mathrm{H}}(\theta) x^{g}[n]
\end{array}\right] .
$$

By explicitly computing the outer product and summing over all transmissions and subcarriers, it is possible to prove that the entries of the FIM scale with $\frac{P}{\sigma^{2}} N G N_{\mathrm{BS}}$, for both UL and $\mathrm{DL}^{1}$. Thus, the CRLB can be approximated as

$$
\mathrm{CRLB}_{\boldsymbol{\eta}}^{\mathrm{UL} / \mathrm{DL}}=\left(\boldsymbol{J}_{\boldsymbol{\eta}}^{\mathrm{UL} / \mathrm{DL}}\right)^{-1} \propto \frac{1}{N G N_{\mathrm{BS}} P / \sigma^{2}} \boldsymbol{A}^{\mathrm{UL} / \mathrm{DL}}
$$

where $\boldsymbol{A}^{\mathrm{UL} / \mathrm{DL}}$ are two matrices independent of $N_{\mathrm{BS}}, G$, and $N$.

The position error bounds (PEBs) can be then computed by adding the third and fourth diagonal entries of the $\mathrm{CRLB}_{\eta}^{\mathrm{UL} / \mathrm{DL}}$ matrix, and taking the square root as $\mathrm{PEB}^{\mathrm{UL} / \mathrm{DL}}=$ $\sqrt{\left[\mathrm{CRLB}_{\boldsymbol{\eta}}^{\mathrm{ULLL}}\right]_{3,3}+\left[\mathrm{CRLB}_{\boldsymbol{\eta}}^{\mathrm{UL} / \mathrm{DL}}\right]_{4,4}}$ where $[\cdot]_{j, j}$ selects the $j$-th diagonal entry of $\mathrm{CRLB}_{\eta}^{\mathrm{ULDL}}$. The analysis above thus reveals that the performance in terms of PEBs will scale by a factor approximately equal to $\sqrt{\lambda}$ when any of the parameters $N, G, N_{\mathrm{BS}}, P / \sigma^{2}$ is multiplied by $\lambda$.

By further inspecting $\boldsymbol{J}_{\boldsymbol{\eta}}^{\mathrm{ULDL}}$ it is possible to show that the entries of the two matrices $\boldsymbol{A}^{\mathrm{UL} / \mathrm{DL}}$ contain the terms $\left\|\boldsymbol{F}^{\mathrm{H}} \boldsymbol{a}_{\mathrm{BS}}\right\|^{2}$ and $\left\|\boldsymbol{F}^{\mathrm{H}} \boldsymbol{B} \boldsymbol{a}_{\mathrm{BS}}\right\|^{2}$ for DL, while the expressions for UL are $\left\|\boldsymbol{a}_{\mathrm{BS}}\right\|^{2}=1$ and $\left\|\boldsymbol{B} \boldsymbol{a}_{\mathrm{BS}}\right\|^{2} \approx N_{\mathrm{BS}}^{2} / 3$, respectively. By the Cauchy-Schwarz inequality, $\left\|\boldsymbol{F}^{\mathrm{H}} \boldsymbol{a}_{\mathrm{BS}}\right\|^{2} \leq\left\|\boldsymbol{a}_{\mathrm{BS}}\right\|^{2}$ (and analogously for the other term) so we conclude that, for the same transmit power $P$, UL provides more information than DL. For the off-diagonal entries (assuming a symmetric spectrum and large $N_{\mathrm{BS}}$ ), it can be shown after some calculation that only the terms relative to $\theta$ and $\phi$ remain, and they are proportional to $\boldsymbol{a}_{\mathrm{BS}}^{\mathrm{H}} \boldsymbol{B} \boldsymbol{a}_{\mathrm{BS}} \approx N_{\mathrm{BS}} / 2$ in UL and $\Re\left\{\boldsymbol{a}_{\mathrm{BS}}^{\mathrm{H}} \boldsymbol{F} \boldsymbol{F}^{\mathrm{H}} \boldsymbol{B} \boldsymbol{a}_{\mathrm{BS}}\right\} \approx$ $N_{\text {BS }} / 2$ in DL. This implies that the FIM in UL and DL behaves essentially in the same way (asymptotically), but with a performance penalty for the DL, which can only be mitigated through the larger DL transmit power or through the beamforming matrix $\boldsymbol{F}$.

\footnotetext{
${ }^{1}$ Details of the proof are omitted due to space limitations. The main assumptions are large $N$, i.i.d. symbols with zero mean and unitary variance, and independence of $\boldsymbol{F}$ and $\boldsymbol{a}_{\mathrm{BS}}(\theta)$ with respect to $n$ and $g$, so that the normalized sums can be approximated by the corresponding expected values.
} 


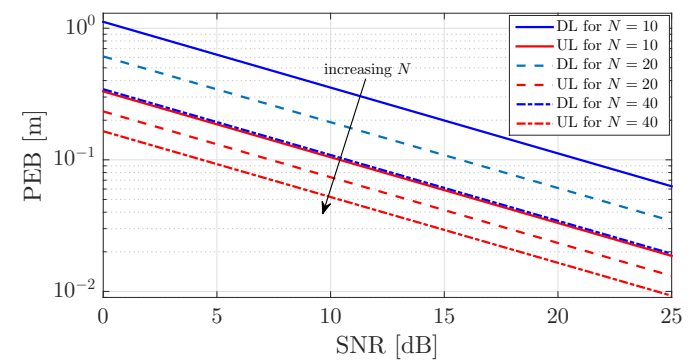

Fig. 1: PEBs in UL and DL for $N_{\mathrm{BS}}=10$ and for a varying $N$ as a function of the SNR.

\subsection{Downlink vs Uplink Localization}

We have seen from the theoretical analysis above that there exists a gap in the achievable accuracy, with the UL exhibiting higher estimation performance than DL, when the transmit power (as well as all the other parameters) are the same.

To confirm this finding, we report numerical results assuming a BS located at known position $\left[\begin{array}{ll}3 & 0\end{array}\right]^{\mathrm{T}}[\mathrm{m}]$ and equipped with $N_{\mathrm{BS}}=10$ antennas, while the MS is placed at $\boldsymbol{p}=\left[\begin{array}{ll}5 & 15\end{array}\right]^{\mathrm{T}}[\mathrm{m}]$, so that the distance between them reflects the realistic coverage expected in $5 \mathrm{G}$ communications [4]. Moreover, $f_{c}=60 \mathrm{GHz}, B=40 \mathrm{MHz}$, and the transmit power $P$ is varied to obtain different SNRs, but always below the maximum value of $1 \mathrm{~W}$ reported in [14] (more precisely, to obtain SNRs between 0 and $25 \mathrm{~dB}, P$ approximately ranges from $1 \mathrm{~mW}$ to $100 \mathrm{~mW}$ ). In absence of knowledge about the MS position, the beamforming matrix $\boldsymbol{F}$ is set to $M$ uniformly-spaced beams. The number of transmissions is $G=1$, and the path loss $\rho$ is computed according to [15]. Finally, the SNR (in dB) is defined as SNR $\stackrel{\text { def }}{=} 10 \log _{10}\left(\frac{P}{\rho N_{0} B}\right)$ with $N_{0} B$ the noise power.

In Fig. 1, we depict the PEBs as a function of the SNR for a varying number of subcarriers $N$. As it can be noticed, the performance gap between UL and DL channels is evident in all the considered cases. Notice that for the considered small values of the parameters the scaling is not exactly $\sqrt{2}$ (especially for DL) and is slightly different between UL and DL. This is visible in Fig. 1 and translates into about $6 \mathrm{~dB}$ gain in DL vs about $4 \mathrm{~dB}$ gain in UL, a difference that anyway disappears for larger $N$ since UL and DL both scale in the same way (as found in the asymptotic analysis in Sec. 3.1). A similar behavior can be observed on the other parameters, confirming that the gap cannot be filled when UL and DL have the same parameters.

However, one should notice that the power budget in UL is typically much lower than that in DL, as better explained later. Moreover, we will show below that the performance in DL can be significantly improved by resorting to adaptive beamforming. In [11], it has been proved that at least two distinct beams are necessary to solve for the unknown MS position using a single-antenna receiver. We start by studying the case
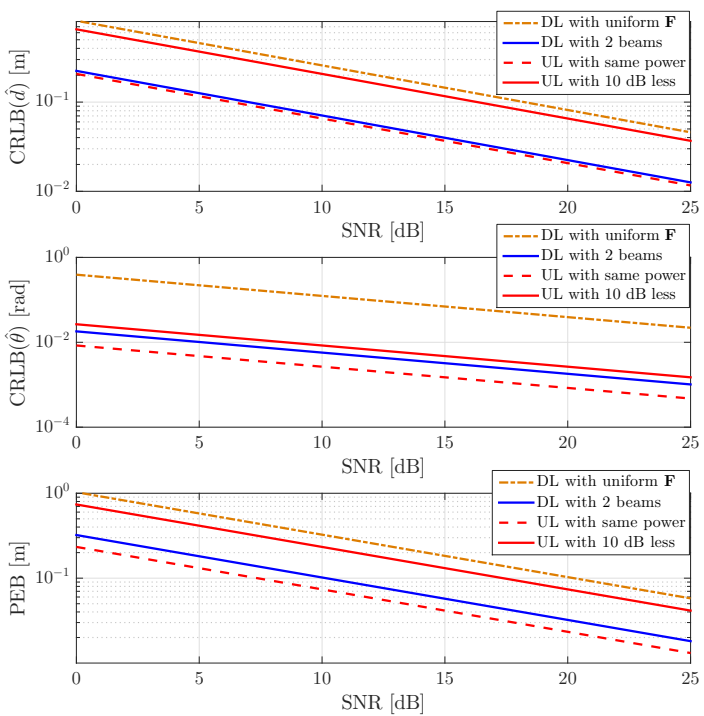

Fig. 2: CRLBs of $d, \theta$ and $\boldsymbol{p}$ as function of the SNR for UL and DL with uniform and adaptive beamforming matrix $\boldsymbol{F}$.

in which the $\operatorname{AOD} \theta$ is perfectly known to the $\mathrm{BS}$, and is used to steer the transmission towards the MS. More precisely, we consider a beamforming matrix ${ }^{2} \boldsymbol{F}=\left[\boldsymbol{f}_{1} \boldsymbol{f}_{2}\right]$ where

$$
\begin{aligned}
& \boldsymbol{f}_{1}=\frac{1}{\sqrt{N_{\mathrm{BS}}}}\left[1 \mathrm{e}^{j \frac{2 \pi}{\lambda} d \sin (\theta+\delta)} \cdots \mathrm{e}^{j\left(N_{\mathrm{BS}}-1\right) \frac{2 \pi}{\lambda} d \sin (\theta+\delta)}\right]^{T} \\
& \boldsymbol{f}_{2}=\frac{1}{\sqrt{N_{\mathrm{BS}}}}\left[1 \mathrm{e}^{j \frac{2 \pi}{\lambda} d \sin (\theta-\delta)} \cdots \mathrm{e}^{j\left(N_{\mathrm{BS}}-1\right) \frac{2 \pi}{\lambda} d \sin (\theta-\delta)}\right]^{T}
\end{aligned}
$$

are the two distinct beams, and $\delta$ is a prefixed beams separation interval; considering that the half-power beamwidth of a ULA is roughly equal to $2 / N$ [16], in the following we consider $\delta=1 / N \approx 5^{\circ}$ so as to have a sufficient overlap of the two beams. It is worth noting that such a design does not require any specific optimization, hence it can be easily implemented once the AOD information is available.

In Fig. 2, we compare the CRLBs of $d=c \tau, \theta$ and $\boldsymbol{p}$ derived on the basis of the FIM analysis in Sec. 3 for $N=20$, as a function of the SNR. We first notice that, in real scenarios, the transmit power in the UL can be as low as $10 \mathrm{~mW}$ [17], a value $20 \mathrm{~dB}$ less than the maximum power in the DL $(1 \mathrm{~W})$. To conduct a fair comparison, in the following we assume that the UL has a transmit power which is $10 \mathrm{~dB}$ less than DL. In this more realistic setup, the theoretical bounds of the UL (solid red curves) significantly increase, but the gap with the DL using a uniform $\boldsymbol{F}$ is still present. Remarkably, adopting the proposed precoding matrix $\boldsymbol{F}$ leads to a dramatic improvement of the DL performance, with accuracy (solid blue curves) that is about $60 \%$ higher than that in the UL (under realistic power assumptions). This behavior can be explained by noting that steering the transmission towards the MS enables

\footnotetext{
${ }^{2}$ Before renormalization to guarantee $\|\boldsymbol{F}\|_{F}=1$.
} 


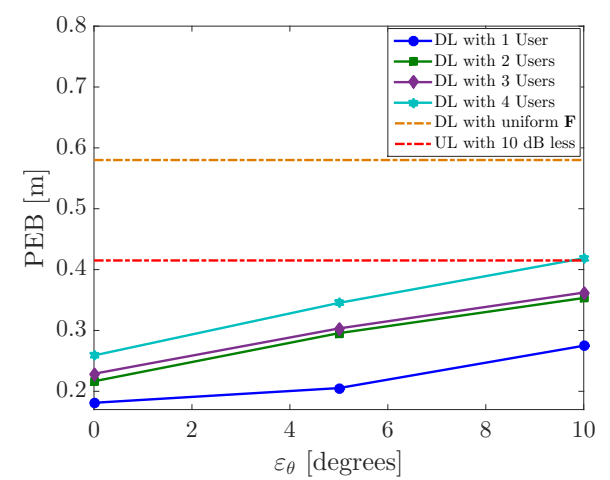

Fig. 3: Theoretical performance as a function of the error $\varepsilon_{\theta}$ for $\mathrm{SNR}=5 \mathrm{~dB}$, also in presence of multiple users.

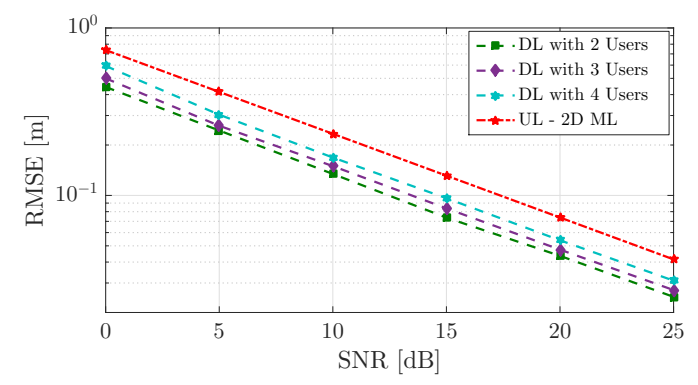

Fig. 4: RMSE of estimated $\boldsymbol{p}$ in DL versus 2D ML estimator in UL (with $10 \mathrm{~dB}$ less power) as a function of the SNR.

a much more accurate estimation of the AOD, with a gain of about $25 \mathrm{~dB}$ compared to the case of uniform $\boldsymbol{F}$. Furthermore, the impact of the beamforming leads to a $10 \mathrm{~dB}$ gain in the estimation of the TOF, resulting in an overall improvement of the PEB. This is an interesting finding since it demonstrates that in practical settings (unequal powers) position estimation in DL can be much more accurate than that in UL, in spite of the fact that the latter exploits a $N_{\text {BS }}$-dimensional vector of samples for each subcarrier (array at the BS).

To complete the analysis, we evaluate the achievable performance assuming that the $\operatorname{AOD} \theta$ is known to the BS up to an estimation error, i.e., $\hat{\theta}=\theta+\varepsilon_{\theta}$. In Fig. 3, we compare the PEBs as a function of $\varepsilon_{\theta}$, for SNR $=5 \mathrm{~dB}$. The obtained results reveal that the MS can be accurately localized in DL even in presence of significant pointing errors (up to $10^{\circ}$ ).

To further inspect the impact of the proposed beamforming, we investigate the achievable performance in presence of other users in the system, i.e., the case in which the BS allocates additional beams in $\boldsymbol{F}$ to serve multiple directions. When at least two users are present, one beam to each user is indeed theoretically sufficient to guarantee a non-singular FIM. However, we opted to allocate two beams for each user as done in (4)-(5) so as to introduce robustness against errors in the pointing directions. From Fig. 3 it emerged that, by using the proposed $\boldsymbol{F}$, the BS can simultaneously serve multiple users while the MS can localize itself with an accuracy always better than the one in the UL, even when the system is close to the maximum number of users that can be served.

\section{PROPOSED APPROACH AND RESULTS}

The theoretical analysis provided so far demonstrates that a very accurate localization can be obtained in the DL when a suitable $\boldsymbol{F}$ is adopted. In this section, we propose a lowcomplexity algorithm that leverages the insights from the analyses in Sec. 3.2 to improve the localization performance. The proposed approach involves a two-step process:

Step 1 The BS broadcasts in DL using a uniform beamforming $\boldsymbol{F}$ (no information about the MS). The MS exploits a two-way protocol to solve for the unknown synchronization offset; then, it computes a first coarse estimate of $\tau$ and $\theta$ through the low-complexity unstructured ML estimator (UML) [11], which only requires two simple 1D searches over the space of $\tau$ and $\theta$.

Step II The MS sends in UL the first coarse estimate of $\hat{\theta}$ back to the BS, which can then adaptively adjust $\boldsymbol{F}$ using two beams steered towards $\hat{\theta}$ (ref. Sec. 3.2). From now on, the MS can compute position updates (using the UML estimator) by exploiting the sole DL channel, benefiting from more directional transmissions.

Fig. 4 reports the RMSE of the proposed algorithm vs. SNR, for a different number of served users (three additional users placed at $10^{\circ}, 25^{\circ}$ and $64^{\circ}$ ). We compare the proposed approach against the $2 \mathrm{D}$ ML estimator for the UL derived in $[18,19]$, which performs an exhaustive 2D search in $(\tau, \theta)$ to estimate $\boldsymbol{p}$. Since it closely attains the corresponding PEB, it can be taken as a benchmark for the UL. As it can be seen, the proposed approach significantly outperforms the ML estimator in the UL, with much lower computational complexity, even in presence of multiple users. In terms of channel utilization, it preserves all the advantages of a DL-only positioning approach, limiting the use of the UL only to the preliminary synchronization phase and to feedback the coarse estimate $\hat{\theta}$ obtained in Step 1.

\section{CONCLUSION}

We have investigated the problem of MS position estimation in mmWave MISO systems. A theoretical comparison between UL and DL has been conducted, showing that a suitable design of the transmit beamforming can make the position estimation in DL much more accurate than in the UL under realistic power assumptions. Based on such analysis, we have proposed a practical two-step algorithm, which exploits adaptive beamforming to provide high accuracy localization in DL, even in presence of multiple users. Remarkably, it preserves all the advantages of DL-only positioning, while enabling localization of MIMO devices even in presence of severe obstructions, if just one antenna is in LOS with a BS. 


\section{REFERENCES}

[1] T. S. Rappaport, S. Sun, R. Mayzus, H. Zhao, Y. Azar, K. Wang, G. N. Wong, J. K. Schulz, M. Samimi, and F. Gutierrez, "Millimeter Wave Mobile Communications for 5G Cellular: It Will Work!," IEEE Access, vol. 1, pp. 335-349, 2013.

[2] R. Di Taranto, S. Muppirisetty, R. Raulefs, D. Slock, T. Svensson, and H. Wymeersch, "Location-Aware Communications for $5 \mathrm{G}$ Networks: How location information can improve scalability, latency, and robustness of 5G," IEEE Signal Processing Magazine, vol. 31, no. 6, pp. 102-112, Nov 2014.

[3] J. A. del Peral-Rosado, R. Raulefs, J. A. López-Salcedo, and G. Seco-Granados, "Survey of cellular mobile radio localization methods: From $1 \mathrm{G}$ to 5G," IEEE Communications Surveys Tutorials, vol. 20, no. 2, pp. 1124-1148, Secondquarter 2018.

[4] M. Koivisto, M. Costa, J. Werner, K. Heiska, J. Talvitie, K. Leppänen, V. Koivunen, and M. Valkama, "Joint Device Positioning and Clock Synchronization in 5G Ultra-Dense Networks," IEEE Transactions on Wireless Communications, vol. 16, no. 5, pp. 2866-2881, May 2017.

[5] A. Hu, T. Lv, H. Gao, Z. Zhang, and S. Yang, "An ESPRIT-Based Approach for 2-D Localization of Incoherently Distributed Sources in Massive MIMO Systems," IEEE Journal of Selected Topics in Signal Processing, vol. 8, no. 5, pp. 996-1011, Oct 2014.

[6] N. Garcia, H. Wymeersch, E. G. Larsson, A. M. Haimovich, and M. Coulon, "Direct Localization for Massive MIMO,” IEEE Transactions on Signal Processing, vol. 65, no. 10, pp. 2475-2487, May 2017.

[7] E. Y. Menta, N. Malm, R. Jäntti, K. Ruttik, M. Costa, and K. Leppänen, "On the Performance of AoA-Based Localization in 5G Ultra-Dense Networks," IEEE Access, vol. 7, pp. 33870-33880, 2019.

[8] Angelo Coluccia, "Crowdsensing networks in the IoT age," Transactions on Emerging Telecommunications Technologies, vol. 30, no. 4, pp. e3621, 2019.

[9] Z. Abu-Shaban, X. Zhou, T. Abhayapala, G. SecoGranados, and H. Wymeersch, "Error Bounds for Uplink and Downlink 3D Localization in 5G Millimeter Wave Systems," IEEE Transactions on Wireless Communications, vol. 17, no. 8, pp. 4939-4954, Aug 2018.

[10] A. Shahmansoori, G. E. Garcia, G. Destino, G. SecoGranados, and H. Wymeersch, "Position and Orientation Estimation Through Millimeter-Wave MIMO in 5G
Systems," IEEE Transactions on Wireless Communications, vol. 17, no. 3, pp. 1822-1835, March 2018.

[11] A. Fascista, A. Coluccia, H. Wymeersch, and G. SecoGranados, "Millimeter-Wave Downlink Positioning With a Single-Antenna Receiver," IEEE Transactions on Wireless Communications, vol. 18, no. 9, pp. 44794490, Sep. 2019.

[12] Zohair Abu-Shaban, Henk Wymeersch, Thushara D. Abhayapala, and Gonzalo Seco-Granados, "SingleAnchor Two-Way Localization Bounds for $5 \mathrm{G}$ mmWave Systems: Two Protocols," CoRR, vol. abs/1805.02319, 2018.

[13] V. Sark, E. Grass, and J. Gutiérrez, "Multi-way ranging with clock offset compensation," in 2015 Advances in Wireless and Optical Communications (RTUWO), Nov 2015, pp. 68-71.

[14] A. Maltsev, R. Maslennikov, A. Sevastyanov, A. Khoryaev, and A. Lomayev, "Experimental investigations of $60 \mathrm{GHz}$ WLAN systems in office environment," IEEE Journal on Selected Areas in Communications, vol. 27, no. 8, pp. 1488-1499, October 2009.

[15] Q. C. Li, G. Wu, and T. S. Rappaport, "Channel model for millimeter-wave communications based on geometry statistics," in 2014 IEEE Globecom Workshops (GC Wkshps), Dec 2014, pp. 427-432.

[16] H.L. Van Trees, Detection, Estimation, and Modulation Theory, Part IV: Optimum Array Processing, WileyInterscience, 2001.

[17] M. Zeng, W. Hao, O. A. Dobre, and H. V. Poor, "Energy-Efficient Power Allocation in Uplink mmWave Massive MIMO With NOMA," IEEE Transactions on Vehicular Technology, vol. 68, no. 3, pp. 3000-3004, March 2019.

[18] M. Wax and A. Leshem, "Joint estimation of time delays and directions of arrival of multiple reflections of a known signal," IEEE Transactions on Signal Processing, vol. 45, no. 10, pp. 2477-2484, Oct 1997.

[19] Georgios B. Giannakis, Petre Stoica, and Yingbo Hua, Eds., Signal Processing Advances in Wireless and Mobile Communications, Volume 2: Trends in Single-And Multi-User Systems, Prentice Hall PTR, Upper Saddle River, NJ, USA, 2000. 